\title{
The lambda (twin peak) sign
}

\author{
Tsun Wai Jeffrey Wu๑, ${ }^{1}$ Yan-Lin Li, ${ }^{2}$ Kin-Hoi Wong ${ }^{2}$ \\ ${ }^{1}$ Li Ka Shing Faculty of Medicine, University of Hong Kong, Hong Kong, China \\ ${ }^{2}$ Department of Radiology, Queen Mary Hospital, University of Hong Kong, K3, 102 Pokfulam Road, Hong Kong, China
}

The lambda sign refers to the triangular projection of chorion between the layers of inter-twin membranes which resembles the Greek alphabet "lambda" (Fig. 1). It is best observed between 10 and 14 weeks of gestation

Fig. 1. The eleventh lowercase Greek alphabet “lambda”.
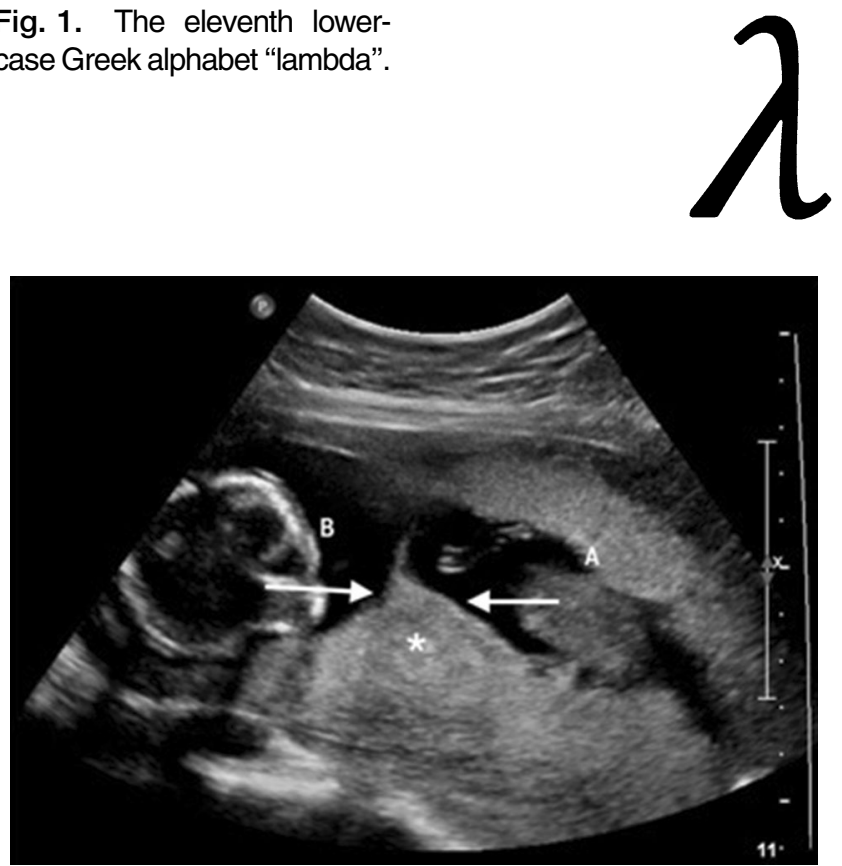

Fig. 2. Transabdominal pelvic ultrasound image obtained at 16 weeks of gestation shows both foeti labeled A and B. The limbs of the lambda sign are highlighted with arrows. The 'peak' is labeled with an asterisk.

on pelvic sonography, and its presence is highly suggestive of dichorionic diamniotic (DCDA) twin pregnancy (Fig. 2). The fused dichorionic placenta also assumes the

Correspondence to: Yan-Lin Li; email: jyl.li@mensa.org.hk

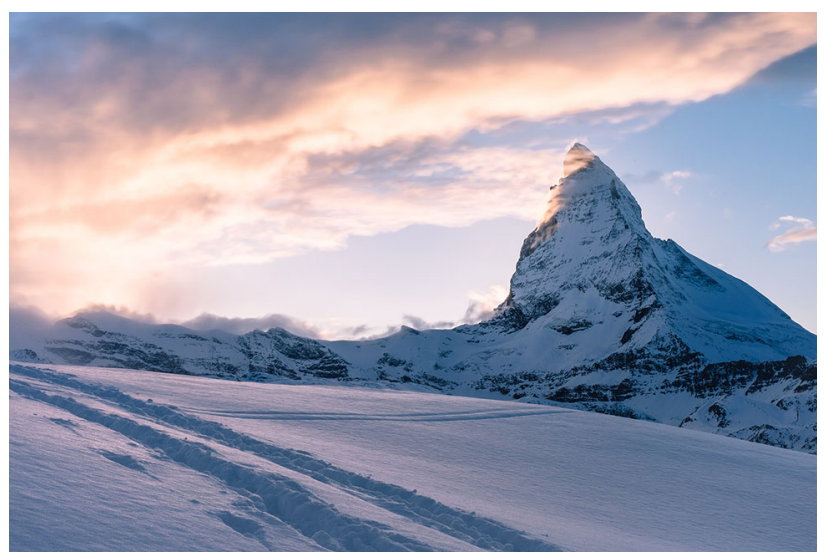

Fig. 3. A peak [2].

appearance of a mountain top between the twin gestational sacs; thus the metaphorical source of its alternate title, the "twin peak" sign (Fig. 3) [1].

Compliance with ethical standards

Funding No funding was received for this study.

Conflict of interest The authors declare that they have no conflict of interest.

Ethical approval This article does not contain any studies with human participants or animals performed by any of the authors.

Informed consent Statement of informed consent was not applicable since the manuscript does not contain any patient data.

\section{References}

1. Sepulveda W, Sebire NJ, Hughes K, Odibo A, Nicolaides KH (1996) The lambda sign at 10-14 weeks of gestation as a predictor of chorionicity in twin pregnancies. Ultrasound Obstet Gynecol 7:421-423. doi:10.1046/j.1469-0705.1996.07060421.x

2. Photo courtesy of free-photos. https://pixabay.com/en/mountainpeak-top-hill-nature-731312/. Accessed 1 Oct 17 